\title{
RELACIÓN ENTRE EL TIPO DE CAMBIO IMPLÍCITO Y EL INDICE DE PRECIOS AL CONSUMIDOR EN VENEZUELA. PERÍODO 1989-2014 ${ }^{1}$
}

\author{
RELATIONSHIP BETWEEN THE IMPLIED EXCHANGE RATE AND \\ CONSUMER PRICE INDEX IN VENEZUELA. PERIOD 1989-2014
}

\author{
Armando Urdaneta ${ }^{2}$, Alberto Castellano ${ }^{3}$, Ronald Prieto ${ }^{4}$ \\ Universidad Nacional Experimental Rafael María Baralt-Venezuela/ \\ Universidad del Zulia-Venezuela/Universidad Simón Bolívar-Colombia
}

RECIBIDO: Septiembre 18 de 2015

ACEPTADO: Noviembre 29 de 2015

\begin{abstract}
RESUMEN
El presente artículo tiene como finalidad analizar la relación que existe entre la variación del tipo de cambio implícito y el índice de precios al consumidor en Venezuela desde 1989 hasta 2014. La investigación fue de tipo documental y correlacional, analítico e interpretativo, basándose en la hermenéutica. Se utilizaron los agregados monetarios del BCV y se analizaron las cien (100) últimas variaciones de índice de precio al consumidor, el tipo de cambio implícito y la correlación existente entre ellas. Se encontró que existe una variación muy similar entre ambas variables estudiadas, con una variación promedio inter trimestral de $7,87 \%$ para la tasa de inflación y de 8,69\% para la tasa de cambio implícito, de acuerdo con el nivel de liquidez monetaria, dividido entre el nivel de reservas internacionales. Las dos variables alcanzaron una correlación lineal de Pearson del 0,93, lo cual denota un alto grado de asociación entre la variación porcentual anual de tipo de cambio y el crecimiento de la tasa de inflación. Todo ello como consecuencia de un incremento de la liquidez monetaria, sin la debida contrapartida en el nivel de reservas internacionales para el caso de la tasa de cambio implícita, con lo cual se genera una devaluación no oficial de la moneda, un incremento general de precios de los productos importados, disminución de los salarios reales de los trabajadores y desequilibrio de la balanza de pagos, debido al aumento de la fuga de capitales por la pérdida de la función de reserva de valor del bolívar. Finalmente, se procedió a realizar el análisis de regresión lineal para construir una función que permita prospectar el comportamiento de la tasa de inflación en función a la variación porcentual de la tasa de cambio implícita. A modo de conclusión, se consiguió evidencia estadística, con los datos y período estudiados, de una correlación positiva entre la tasa de inflación real y la pronosticada.
\end{abstract}

Palabras clave: Política cambiaria, tipo de cambio, índice de precio al consumidor.

\section{ABSTRACT}

This article aims to analyze the relationship between the change in the implicit exchange rate and the consumer price index in Venezuela from 1989 to 2014. The research was correlational documentary, analytical and interpretative, based on hermeneutics ; using monetary aggregates BCV, analyzing the one hundred (100) last variations price index consumer, the implicit exchange rate and the correlation between them, observing as there is a similar variation between the two variables, with an average variation inter quarterly $7.87 \%$ for the inflation rate and $8.69 \%$ for implicit exchange rate, according to the level of monetary liquidity, divided by the level of international reserves, both variables having a linear Pearson correlation of 0.93 ; which denotes a high degree of association between annual percentage change in the exchange rate and the growth of the inflation rate. All this as a result of an increase in monetary liquidity, without the contribution due to the level of international reserves for the case of the exchange rate means, whereby an unofficial currency devaluation is generated, a general price increase of imported products, declining real wages, imbalance in the balance of payments, due to increased capital flight by the loss of function of value of the Bolivar. Finally we proceeded to perform linear regression analysis to build a function to prospect the behavior of the inflation rate based according to the percentage change in the implicit rate of change, where Concluding statistical evidence is obtained, with data and period studied, a positive correlation between the rate of actual inflation and predicted.

Keywords: exchange rate policy, exchange rate and consumer price index.

Este artículo se puede referenciar

Urdaneta, A., Castellano, A. \& Prieto, R. (2016). Relación entre el tipo de cambio implícito y el indice de precios al consumidor en venezuela. Período 1989-2014. En Desarrollo Gerencial Revista de la Facultad de Ciencias Económicas Administrativas y Contables de la Universidad Simón Bolívar-Colombia, 8(1), 49-70.

1 Este artículo constituye un avance del proyecto de investigación "Relación entre el tipo de cambio implícito y el indice de precios al consumidor en Venezuela. Período 1989-2014".

2 Ingeniero en Computación egresado de la Universidad Rafael Belloso Chacín (1998). Magister en Gerencia Empresarial, Magister en Telemática. Doctor en Ciencias Gerenciales. Profesor universitario asociado a dedicación exclusiva del Programa de Administración de la Universidad Nacional Experimental Rafael María Baralt.

2. Economista egresado de la Universidad del Zulia (Luz) (1996). Magíster en Gerencia de Empresas mención Gerencia Financiera. Profesor Asociado a dedicación exclusiva de la Facultad de Ciencias Económicas y Sociales Universidad del Zulia. Investigador del Instituto de Investigaciones Fces Luz. Miembro del PEI. PhD en Economía de Luz.

3. Posdoctor en Gerencia de las Organizaciones (URBE-Venezuela). Doctor en Ciencias Gerenciales (URBE- Venezuela). Magíster en Gerencia Empresarial (URBE- Venezuela). Economista (LUZ, Venezuela). Profesor de Planta, editor de la Revista Desarrollo Gerencial y Decano de la Facultad de Administración y Negocios de la Universidad Simón Bolívar. Barranquilla-Colombia. Investigador adscrito al Centro de Investigación, Desarrollo, Innovación y Tecnología Empresarial (CIDITEC). Email: ronaldprieto1@hotmail.com; rprieto1@unisimonbolivar.edu.co 


\section{INTRODUCCIÓN}

La relación entre la política cambiaria y la inflación constituye uno de los temas más polémicos. En esta investigación se estudia el comportamiento de la tasa de cambio implícita, el índice de precio al consumidor desde el período de 1989 hasta el 2014 y la inflación en Venezuela. Se plantea de antemano que los esquemas de fijación convencional como herramienta antiinflacionaria, tal como el que mantuvo Venezuela hasta febrero de 1983, no son sostenibles a menos que exista un compromiso fiscal que equilibre intertemporalmente las cuentas públicas.

De hecho, los esquemas de control de cambios, aplicados en situaciones de emergencia para contener las salidas de capital y depreciación del tipo de cambio, lejos de contribuir a la solución del problema que pretendieron resolver lo posponen y lo agudizan, en razón de que no atacan las causas últimas de la caída de la demanda por activos internos. Lo anterior, aunado a la falta de libertad en el mercado y a los controles en las importaciones de materia prima, han generado una economía de importación en Venezuela, opuesta a la estimulación del aparato productor nacional, que según Adam Smith es la manera de generar riqueza (citado en Ekelund y Hébert, 2008).

En el presente estudio se utiliza una relación de regresión lineal con la finalidad de construir una función, en este caso lineal, que nos permita prospectar el comportamiento de la tasa de inflación en función a la variación porcentual de la tasa de cambio implícita. A modo de conclusión, con los datos y período estudiados, se consigue evidencia estadística de una correlación positiva entre la tasa de inflación real y la pronosticada, así como su repercusión en la disminución de los salarios reales y en los desequilibrios de la balanza de pagos.

\section{Enfoque epistemológico}

El enfoque económico de Adam Smith, (Herrerías, 2011) parte de la afirmación de que la fuente de la riqueza se encuentra en el trabajo; su sistema caracterizaba las actividades de la agricultura, la manufactura y el comercio, el intercambio viene dado por el uso del dinero y la producción se caracteriza por la división de trabajo. Su análisis se centra en tres variables principales: la división del trabajo, el análisis del precio y de la asignación, y la naturaleza del crecimiento económico. Según el mismo Herrerías (2011), Adam Smith es el primer economista que se refiere a la división del trabajo con un criterio moderno, al plantear que el aumento de la producción es el origen de las riquezas de las naciones, y que el crecimiento económico, que es el aumento continuo de la producción de bienes y servicios que se generan en una sociedad, incrementa a su vez las riquezas en naciones donde existen sistemas que gozan de libertad natural. 
En tal sentido, se puede observar que en Venezuela la vigencia de un control cambiario desde el año 2003, caracterizado por el control del estado sobre las divisas y la desconfianza generado por este, no fomenta el desarrollo de la industria productora de manufacturas. Siguiendo también a a Smith, Ekelund y Hébert (2008) reconocen que incrementar sus riquezas, es la tendencia natural en una sociedad que fomenta el desarrollo continuo en la productividad de su trabajo, generando un mejor resultado en el mismo número de trabajadores.

En palabras de Smith:

El precio del mercado de cualquier mercancía se regula por la proporción entre la cantidad que se lleva al mercado y la demanda de aquellos que están dispuestos a pagar el precio natural de la mercancía o el valor total de la renta. Tales personas pueden ser denominadas demandantes efectivos, y su demanda la demanda efectiva, puesto que es suficiente para llevar al mercado dicha mercancía. Esta demanda es distinta de la demanda absoluta. Un hombre muy pobre puede demandar, en cierto sentido, un carruaje con seis caballos, podría gustarle tenerlo, pero su demanda no es unas demanda efectiva, ya que la mercancía nunca puede ser llevada al mercado para satisfacerle (Citado por Ekelund y Hébert, 2008)

Por esto Smith afirma que si la cantidad de productos disponible en el mercado es menor que la demanda efectiva, no se puede satisfacer a todos los dispuestos a pagar por el valor total de la renta, salarios y beneficios para comprar esta. Más bien, en tal caso, un grupo preferiría pagar un valor mayor para obtenerla, generando esto un precio superior en el mercado; en tanto que si, por el contrario, existe un excedente a la demanda efectiva, no se podría vender toda esta mercancía al valor de la renta, salarios y beneficios, y se tendría que vender a un valor menor del precio natural.

Smith habla de una rivalidad existente entre los compradores y los vendedores del mismo producto, donde el precio del mercado debe cubrir los costos de producción a largo plazo, y quiso elegir el trabajo como el denominador común en el valor, pues, consideraba al precio natural no solo como un precio de equilibrio sino como un patrón invariable en el tiempo. Al respecto, Heilbroner y Milberg (2010) señalan que la tesis monetarista de Friedrich Von Hayek, premio nobel de economía 1974, atribuye a los bancos centrales la facultad de mantener la demanda de capital real dentro de los límites fijados por la oferta de ahorro, o bien mantener el nivel de precios estables; pero no pueden hacer las dos cosas a la vez. Por ello, el objetivo de la política económica no debe ser la estabilidad del nivel de precios sino la estabilidad de la cantidad de dinero en circulación.

Precisamente, por lo antes expuesto, la política monetaria en Venezuela ha generado una distorsión en 
el mercado, producto del crecimiento desproporcionado de la masa monetaria durante el período sujeto a estudio, como se observa en el la Tabla 1, impactando la función del bolívar como reserva de valor, como consecuencia, a su vez, de un aumento desmedido de los precios, debido al desequilibrio existente entre el crecimiento de la oferta monetaria y la renta real de la economía. Todo ello acarrea, además, escasez de materias primas para la producción, debido a la falta de divisas para importar y cubrir la insuficiencia de bienes básicos debido a que el crecimiento de la demanda agregada interna producto de la expansión monetaria es muy superior a la capacidad de la economía para satisfacerla y a que en realidad no se cuenta con las divisas suficientes para satisfacerlas por la vía de la importaciones. A esto se suma la fuga de capitales ya que, en tales circunstancias, los agentes económicos buscan preservar el valor de sus capitales colocándolos en una moneda más fuerte en el exterior.

Ahora bien, según el BCV (2014) la política cambiaria se puede definir como el mecanismo mediante el cual se fija el tipo de cambio, lo cual es una variable estratégica y no sólo un precio más de la economía, ya que genera una señal importante para determinar la estructura económica del país. Sin embargo, es importante destacar que la política de control de cambio en Venezuela no ha tenido ni tendrá el resultado que se busca mientras no se acompañe de políticas fiscales restrictivas que disminuyan el gasto corriente y el déficit fiscal, . En efecto, más allá del aumento de las tasa de interés, coeficiente de caja, tasa de redescuento del Banco Central como últimos prestamistas o la emisión de títulos de estabilización monetaria, debe controlarse la cantidad de dinero circulante en la economía, como se ha evidenciado en el período sujeto a estudio y se muestra en la Tabla 1.

Tabla 1. Comportamiento de la liquidez monetaria en el período 1989 - 2014

\begin{tabular}{|c|c|c|}
\hline Año & $\begin{array}{c}\text { M2 (Liquidez Monetaria) } \\
\text { Bolívares fuertes }\end{array}$ & $\begin{array}{c}\text { Variaciones Porcentuales Anuales de M2 } \\
\text { (Liquidez Monetaria) Bolívares fuertes }\end{array}$ \\
\hline 1.989 & 463.816 .000 & $38,59 \%$ \\
\hline 1.990 & 746.477 .000 & $60,94 \%$ \\
\hline 1.991 & 1.116 .103 .000 & $49,52 \%$ \\
\hline 1.992 & 1.321 .261 .000 & $18,38 \%$ \\
\hline 1.993 & 1.660 .677 .000 & $25,69 \%$ \\
\hline 1.994 & 2.570 .250 .000 & $54,77 \%$ \\
\hline 1.995 & 3.548 .277 .000 & $38,05 \%$ \\
\hline 1.996 & 5.391 .452 .000 & $51,95 \%$ \\
\hline 1.997 & 8.945 .741 .000 & $65,92 \%$ \\
\hline 1.998 & 10.621 .846 .000 & $18,74 \%$ \\
\hline 1.999 & 12.734 .324 .000 & $19,89 \%$ \\
\hline
\end{tabular}




\begin{tabular}{|l|c|c|}
\hline 2.000 & 16.284 .578 .000 & $27,88 \%$ \\
\hline 2.001 & 16.976 .436 .000 & $4,25 \%$ \\
\hline 2.002 & 19.573 .369 .000 & $15,30 \%$ \\
\hline 2.003 & 28.569 .243 .000 & $45,96 \%$ \\
\hline 2.004 & 42.493 .491 .000 & $48,74 \%$ \\
\hline 2.005 & 66.070 .833 .000 & $55,48 \%$ \\
\hline 2.006 & 111.672 .650 .000 & $69,02 \%$ \\
\hline 2.007 & 147.010 .616 .000 & $31,64 \%$ \\
\hline 2.008 & 187.631 .161 .000 & $27,63 \%$ \\
\hline 2.009 & 249.098 .076 .189 & $32,76 \%$ \\
\hline 2.010 & 296.595 .794 .452 & $19,07 \%$ \\
\hline 2.011 & 446.616 .842 .000 & $50,58 \%$ \\
\hline 2.012 & 719.047 .478 .000 & $61,00 \%$ \\
\hline 2.013 & 1.220 .136 .557 .000 & $69,69 \%$ \\
\hline 2.014 & 2.001 .114 .005 .000 & $64,01 \%$ \\
\hline
\end{tabular}

Fuente: Banco Central de Venezuela (2015)

En razón de ello, Fernández, Rodríguez y Parejo (2008) definen la política monetaria como la influencia del Banco Central sobre la oferta monetaria, las tasas de interés y las condiciones financieras de la economía. Su objetivo final es preservar el poder adquisitivo de la moneda mediante la creación de condiciones monetarias y financieras que favorezcan la estabilidad de precios. Los principales instrumentos de la política monetaria son las operaciones de mercado abierto, los requisitos de encajes bancarios y los créditos a los bancos.

En este sentido, para Blanchard (2006) el índice de precios nacional del consumidor se define como un índice de precios al consumidor referido a la totalidad del país. Que no es más que la variación de precio, donde se mide el porcentaje de incremento del costo de una canasta básica de bienes adquirida en un período de tiempo determinado, adquirida por el consumidor. Según Mochón (2008), la tasa de cambio o tipo de cambio es el precio de una moneda expresado en otra. El tipo de cambio se traduce, con otras palabras, como la cantidad de unidades de la moneda nacional por unidad en relación con la moneda extranjera. Los tipos de cambio son una fuente útil de información que orienta las operaciones internacionales de bienes, servicios y capital. Justamente, las relaciones entre las monedas de mayor utilización en el mercado internacional son publicadas diariamente para que el público sepa como se intercambian entre sí y, en la actualidad, obviamente el dólar es la divisa más importante que se emplea como referencial para la medición del valor de las restantes monedas. 
El sistema de tipos de cambio entre divisas, según Fernández, Rodríguez y Parejo (2008), nace de la necesidad de divisas extranjeras por parte de empresas y ciudadanos nacionales. En otras palabras, el tipo de cambio proviene de la circulación de capital, bienes, servicios y personas entre fronteras, esto es, desde de la existencia de comercio internacional. En Venezuela, la fijación del tipo de cambio oficial tiene que ver con el establecimiento del precio legal de las divisas, el cual es determinado por el gobierno (no por el mercado). Tal como sucede en la mayoría de los países petroleros y economías pequeñas, en Venezuela se ha optado históricamente por la socialización del riesgo cambiario en parte porque la fijación del tipo de cambio permitía a las autoridades contener la volatilidad cambiaria resultante de la actividad petrolera.

La política cambiaria en Venezuela viene dada por 4 tasas de cambio, donde tenemos: un dólar oficial en 6,3 bs por dólar; otro sistema complementario de divisas fluctuante en 11,3, aproximadamente; un segundo sistema complementario de divisas con una banda de 49.93 bs hasta 55bs por dólar, que es en sí un dólar implícito; y un dólar paralelo o mercado negro cercano a los 90 bs por dólar.

Con el control cambiario, el gobierno privilegia la importación y la especulación en detrimento de la producción y, así, tarde o temprano se hace inevitable la devaluación. Para estabilizar el tipo de cambio de forma sostenida, como sucedía antes del viernes negro, es esencial estabilizar primero la inflación, lo cual reclama mayor flexibilidad en la producción, mayor inversión, seguridad jurídica, política y económica y sobre todo un arbitraje imparcial por parte del Estado, pues el intento fallido del gobierno de alterar la distribución primaria del ingreso por medio de la sobrevaluación cambiaria ha estimulado las importaciones y desincentivado la producción nacional. Todos estos factores de riesgo disminuyen la inversión extranjera, las reservas internacionales y aumentan los precios.

Finalmente, es de suma importancia que el Estado venezolano adopte políticas públicas que estén articuladas entre sí, entre ellas las políticas fiscales, conjuntamente con las políticas sociales, monetarias y de producción, para lograr la consecución de los objetivos macroeconómicos, generando niveles de bienestar en la población venezolana. Puesto que en la medida, en que se desarrolle el sector productivo del país, a través de incentivos a la producción para generar la elevación del empleo en la población, se controle el proceso inflacionario a través de la preservación del valor de la moneda, y se apliquen estrategias de crecimiento, en esa medida, se estabilizará la economía en un período sostenido.

\section{Teoría cuantitativa del dinero}

Según Ekelund y Hébert (2008), la falta de fe en la política monetaria como mecanismo estabilizador central en la macroeconomía se mantuvo hasta bien entrados los años sesenta. Sin embargo, las sugerencias keynesianas sobre el gasto con déficit fueron seguidas fácilmente durante los períodos de recesión, mientras 
que los presupuestos con superávit o equilibrados durante los períodos de inflación han sido sumamente raros y muy impopulares dentro de la clase política. En otras palabras, los mismos principios keynesianos que convirtieron la economía en una economía a prueba de deflación o depresión pueden haberla inclinado a la inflación.

Tales incrementos de dicho stock acarrearon problemas graves y persistentes de inflación. Como era de prever, estos acontecimientos de las últimas décadas han llevado a una confrontación con la economía keynesiana y a un real y practico resurgimiento del interés por el "monetarismo", que no es otra cosa que un refinamiento de la teoría cuantitativa neoclásica. (En términos teoricos, el monetarismo en forma de teoría cuantitativa nunca estuvo ausente de la escena intelectual de la economía).

Ekelund y Hébert (2008) también arguyen que, con unas pocas y notables excepciones, como las de Locke y Thornton, ningún autor asignó un papel explícito al tipo de interés como determinante importante de la actividad económica. Por otra parte, la teoría cuantitativa no era puramente mecánica, puesto que los aumento de la cantidad de dinero fueron vistos por Cantillon, Thornton, Ricardo y Mill como algo que influía en la demanda de mercancías y, a través de una mayor demanda, elevaba los precios. Los autores clásicos discutieron a menudo las fuerzas que conservarían (o destruirían) esos nuevos equilibrios. Una gran parte de este vacío lo llenaron en un principio los autores neoclásicos Irving Fisher y Knut Wicksell.

En este sentido, en 1911, el profesor de la Universidad de Yale Irving Fisher (1867-1947) fue un poco más allá que Jhon Stuart Mill y dedujo un marco matemático para exponer las conclusiones de la teoría cuantitativa. Fisher escribió: $M V+M^{\prime} V^{\prime}=P T$, donde $M$ es el stock de dinero en circulación; V es la velocidad anual de circulación del dinero, o sea, la proporción en que el dinero cambia de manos; M' es el volumen de los depósitos a la vista en los bancos; V' es la velocidad anual de circulación de lo físico de las transacciones. Como que nuestra definición moderna de dinero incluye los depósitos bancarios a la vista, la ecuación anterior puede reescribirse de forma más simple como MV = PT, que en el futuro se conocería como ecuación de cambio Fisher.

Fisher se dio cuenta de que su ecuación de cambio era una identidad contable y, por tanto, una perogrullada. Pero esto no la invalida desde el punto de vista de la teoría económica. De hecho, Fisher la utilizo para afirmar una vez más la proporcionalidad existente entre los aumentos de $\mathrm{M}$ y los aumentos de $\mathrm{P}$. La ecuación de cambio, bajo ciertos supuestos, se convirtió a continuación en una expresión matemática de la teoría cuantitativa. Los supuestos de Fisher eran que la velocidad (V) y el volumen de la actividad (T) eran independientes de la oferta monetaria y que el nivel de precios era una variable mas pasiva que activa. De aquí que pudiera afirmar y afirmase, la estricta proporcionalidad entre M y P como fenómeno a largo plazo. 
Ahora bien, según Ekelund y Hébert (2008), más importante que su interpretación matemática de la teoría cuantitativa estricta, es la identificación lograda por Fisher, de la relación entre un aumento de la cantidad de dinero y el subsiguiente aumento de los precios. El vínculo perdido (que asegura la estabilidad del equilibrio monetario) es el efecto de saldos reales Y puede explicarse de esta manera: Un aumento de las tenencias monetarias de los individuos altera la relación óptima entre los saldos en efectivo y los gastos de los individuos.

En términos walrasianos, una mayor cantidad de dinero al nivel de precios existentes genera un exceso de oferta de saldos monetarios en manos de los individuos. Así pues, estos intentan reducir su exceso de saldos monetarios aumentando los gastos. Además, si el producto permanece invariable como Fisher suponía), el aumento de la demanda de dinero elevara los precios hasta que alcancen la misma proporción que el aumento de dinero. De esta manera se alcanza y se mantiene un nuevo equilibrio, porque los saldos monetarios de los individuos han regresado a su nivel óptimo.

Fisher no explotó por completo el efecto saldos reales. Por ejemplo, nunca demostró cómo podía utilizarse el exceso de saldos monetarios para adquirir valores, elevando de este modo la cotización de dichos valores y disminuyendo el tipo de interés. En otras palabras, Fisher no demostró nunca cómo un aumento del dinero podía producir indirectamente un aumento del producto, a través de unos tipos de interés más bajos (aunque Wilcksell fue más lejos al llenar esta laguna, como veremos). En su lugar, Fisher volvió a la interrelación existente entre la inflación, tipos de interés, expectativas y tenencias de saldos reales en efectivo.

Así, unas tasa de expansión monetaria más altas pueden llevar, inicialmente, a unos tipos de interés nominal más bajos (a través de un aumento de la oferta de fondo prestables), pero con el tiempo, los precios más altos llevan, a través de las expectativas inflacionistas, a aumentos del tipo nominal y una inflación más alta. Este principio, que figura en el repertorio de los modernos monetaristas, fue uno de los mayores descubrimientos de Fisher. Volveremos sobre estos temas, pero ahora consideraremos las contribuciones de Wicksell al paradigma monetarista.

\section{¿Qué es la política monetaria?}

Según el BCV (Banco Central de Venezuela, 2015), son decisiones que dicho ente toma para influir sobre la oferta monetaria, las tasas de interés y las condiciones financieras de la economía su objetivo final es preservar el poder adquisitivo de la moneda mediante la creación de condiciones monetarias y financieras que favorezcan la estabilidad de precios. Los principales instrumentos de la política monetaria son las operaciones de mercado abierto, los requisitos de encajes bancarios y los créditos a los bancos. 
Los objetivos de la política monetaria consisten en controlar la oferta monetaria y disponibilidad de dinero acorde con el nivel de actividad económica con el objeto de impedir desequilibrios que puedan alentar procesos inflacionarios, y esto puede lograrse a través del tipo de interés con el propósito de mantener la estabilidad de precios, aumento el costo del acceso al crédito(Guerra, 2013). Por otro lado, Mochón (2008) afirma que en la medida que la política monetaria crea y mantiene condiciones monetarias y crediticias favorables a la estabilidad de precios, motiva el desarrollo de iniciativas privadas en inversión y consumo que aceleran el ritmo de la actividad económica; lo cual es un requisito fundamental para un crecimiento económico sostenido a largo plazo.

En este sentido las medidas antes señaladas, ayudaran a la estabilidad de la tasa de cambio, lo cual contribuye con el objetivo general de equilibrar la balanza de pagos, asegurar la continuidad de los pagos internacionales del país y mantener la competitividad externa de la producción doméstica. Es por ello que el equilibrio en el mercado de dinero se alcanza en un punto en el cual la cantidad que los individuos desean demandar de saldos reales es igual a la ofrecida por el Banco Central. Es decir, debe existir un equilibrio entre la tasa de crecimiento de la liquidez monetaria y la tasa de crecimiento de la renta real. Dado un nivel de precios, el Banco Central sigue una política monetaria expansiva si incrementa la oferta haciendo que el tipo de interés se reduzca. Por el contrario, si se aplica una política monetaria contractiva, la oferta se reduce elevando el tipo de interés.

En el caso venezolano se ha seguido una política monetaria expansiva desde comienzos del año 1983, cuando nuestra economía comenzó a presentar problemas de déficit fiscal para financiar precisamente dicho déficit, donde la tasa de crecimiento nominal del dinero ha sido muy superior a la tasa de crecimiento de la renta real; especialmente a partir del año 2004 con un crecimiento hasta finales del 2014 del 3777\%; no así en los períodos 1984-1993 donde la liquidez monetaria solo creció 907\% y en el período 1994-2003 donde dicha variable conocida como oferta monetaria creció 1405\% según estimaciones mediante los informes económicos del Banco Central de Venezuela (2014).

\section{MÉTODO}

Por las características de la investigación, se clasifica como descriptiva, ya que se orienta a recolectar información relacionada con el estado real de personas, objetos, situaciones o fenómenos, tal cual como se presentan en el momento de su recolección (Chávez, 2001). El presente estudio también basó sus aspectos metodológicos, a través de una investigación de tipo documental bibliográfica y correlacional, por cuanto se generan análisis críticos y reflexiones que permitan determinar la relación entre la tasa de cambio implícita en el índice precios al consumidor en la economía venezolana, se considera además de tipo cuali-cuantita- 
tiva, al ser desarrollada bajo el método de la hermenéutica según (Martínez, 2013), la cual es considerada como la ciencia de interpretación del lenguaje de los autores, en el caso particular de este estudio conocer a través de un análisis exhaustivo el comportamiento del índice de precios al consumidor en los últimos 25 años.

En este sentido, la investigación se ubicó dentro del diseños no experimental puesto no se manipula la variables y se analizan en su estado natural; y en el diseño transeccionales descriptivos, los cuales tienen como objetivo indagar la incidencia y los valores en que se manifiestan una o más variables, ubicar, categorizar y proporcionar una visión de una comunidad, evento, contexto, fenómeno o situación (Hernández, Fernández y Baptista, 2008). El procedimiento consiste en medir o ubicar a un grupo de personas, objetos, situaciones, contextos fenómenos, en una variable o concepto y proporcionar su descripción.

Indica la misma fuente que: "Los estudios correlaciónales miden las dos o más variables que se pretenden ver si están o no relacionadas en los mismos sujeto y después se analizan la correlación... la utilidad y el propósito principal de estos estudios... es saber cómo se puede comportar un concepto o variable conociendo el comportamiento de otras variables relacionadas". De igual manera, de acuerdo a las estrategias empleadas en la recolección de datos, el estudio se ubicó en esta clasificación, donde se evaluara la incidencia de las medidas cambiarias adoptadas por el gobierno sobre el proceso inflacionario. En este sentido, para los efectos de esta investigación, se tomó una población formada por todas las medidas cambiarias adoptadas por el Ejecutivo Nacional.

\section{RESULTADOS}

\section{Trayectoria de la política cambiaria 1989-2014}

Según Guerra y Pineda (2000), la política cambiaría en Venezuela asumida por el Banco Central de Venezuela (BCV), en el período sujeto a estudio, ha estado constituida por diferentes sistemas de cambio, antecedido por un período de control de cambios entre febrero de 1983 y febrero de 1989, el cual a su vez fue producto de la crisis en la balanza de pagos que se gestó entre 1979-1982, combinados con altos niveles de inflación, disminución de las reservas internacionales, centralización de los activos externos de Petróleos de Venezuela S.A (PDVSA) y el fondo de inversiones de Venezuela (FIV), en el BCV. Así como la revalorización de las tenencias de oro para fortalecer las reservas internacionales.

Sin embargo debido a la aplicación de políticas fiscales y monetarias de carácter expansiva, le restaron credibilidad al sostenimiento del tipo de cambio fijo, lo cual desató un ataque especulativo durante el segundo semestre de 1982 que culminó en el colapso del arreglo cambiario, una vez que el BCV había perdido más del 40\% de su acervo de reservas internacionales. 
Razón por la cual, durante 1983, se instala un control de cambio cuya política económica consistió en una combinación de expansión fiscal con laxitud monetaria para evitar una mayor contracción económica y preservar los niveles de empleo BCV (1983). De esta manera, según Guerra y Pineda (2000), los objetivos antiinflacionarios y de restauración del equilibrio de las cuentas externas, se remitieron a la imposición de medidas administrativas de racionamiento de divisas y control de precios.

Ahora bien, entre 1983 y 1985, dicho período cambiario se caracterizó por medidas de ajuste encaminadas a eliminar el déficit de la gestión financiera del sector público, mejorar la competitividad de la economía y mitigar el impacto de la devaluación sobre los precios. Ello implicó una devaluación del 41\% de la moneda y un superávit fiscal de 2,1\% del Producto Interno Bruto Real (PIBR) en 1984.

Sin embargo, producto del poco dinamismo que mostraba la economía la cual apenas lograba salir del ciclo de recesión, durante el lapso de 1986-1988, nuevamente se adoptan medidas de política fiscal y monetaria de carácter expansivo para reactivar la economía, pero debido a la disminución de los precios de petróleo en un 47\%, el BCV se vio obligado a devaluar la moneda en un $132 \%$, dando como resultado una tasa de inflación del $35,5 \%$ al cierre de 1988, precedida de 40,3\% en 1987, un déficit en la cuenta corriente de la balanza de pagos de (5.809) millones de dólares y un déficit fiscal de 9,3\% del PIBR, lo cual permitía anticipar un colapso de la balanza de pagos a finales de 1988 con el consecuente cambio de régimen.

El cual se materializó en febrero de 1989 cuando se instrumentó un programa de ajuste con el Fondo Monetario Internacional (FMI), que implicó la adopción de un esquema de flotación del tipo de cambio, el cual fue apoyado con medidas restrictivas de política monetaria y fiscal encaminadas a moderar los efectos inflacionarios del levantamiento de los controles cambiarios y de precios, provocando una contracción de la base monetaria y liquidez monetaria propiciada por el BCV mediante un alza importante de las tasas interés. La mejora sustancial de las cuentas fiscales es atribuible fundamentalmente a las ganancias de ingresos que produjo una devaluación del $(10,4 \%)$ del PIBR y en menor medida una reducción del gasto público.

No obstante, el mercado retardó en la aprobación de leyes fiscales que restringieron las posibilidades de emprender un ajuste fiscal como el que se quería, descargando entonces en la política monetaria todo el peso del ajuste, mediante el aumento permanente de las tasas de interés, dando como resultado al cierre de 1989 que la economía mostrara una alta tasa de inflación conjuntamente con una fuerte recesión causada por el shock de oferta que representó la corrección instantánea de los ajustes cambiarios y la consiguiente devaluación.

Esta situación, según Guerra y Pineda (2000), produjo un viraje de la política fiscal en 1990 para esti- 
mular nuevamente el crecimiento económico en tanto que la política monetaria conservaba su carácter contractiva buscando controlar las presiones inflacionistas, lo que propició un crecimiento del PIBR al cierre de 1992 del 6,1\% con una tasa de inflación que disminuyó del 86,4\% en 1989 al 31,4\% al cierre de 1992, sin embargo la cuenta corriente de la balanza de pagos se mostraba deficitaria en (3.365) millones de dólares, sumado a un déficit fiscal del $(5,8 \%)$ del PIBR, provocando el deterioro de la cartera de crédito del sistema financiero debido a la disminución de la misma.

Por ello en octubre de 1992 ocurre un viraje en la política cambiaria mediante la adopción de un esquema de minidevaluaciones para permitir un deslizamiento suave del tipo de cambio que preservara la competitividad del sector transable, sin embargo, durante 1993 se requirió que el BCV interviniera activamente en el mercado monetario para mantener la estabilidad de la cotización, pero producto de la incertidumbre a principio de 1994, como consecuencia de la crisis financiera y la falta de decisiones en materia de política económica, repercutió sobre las cuentas externas y la situación fiscal lucia muy comprometida como resultado de la disminución de los precios del petróleo y a la suspensión de la aplicación del IVA haciendo las cuentas fiscales deficitarias.

En este sentido, los efectos sobre la economía de las tasas de interés reales positivas produjo el cierre del segundo banco comercial lo cual desencadenaría en una crisis bancaria que sumado al financiamiento monetario de la crisis financiera, propiciaron una corrida de los depósitos que cuestionó la capacidad del BCV para defender el sistema de minidevaluaciones. Como resultado de la crisis financiera, potenció la demanda de activos externos y una pérdida de activos en reserva de 3.790 millones de dólares, equivalente al 45\% del acervo total, lo cual llevó a la aplicación del control de cambio en julio de 1994 hasta julio de 1996, con una devaluación del 23\% respecto al tipo de cambio promedio de mayo de 1994; sin embargo, dadas las dificultades para la obtención de divisas al principio, el mercado paralelo fue adquiriendo cada vez más importancia reflejándose en el diferencial cambiario de $47 \%$ en el segundo trimestre de 1995 al 156\% en el primer trimestre de 1996, haciendo evidente el agotamiento del sistema de control de cambio.

Es entonces, a partir de julio de 1996 cuando se asume el sistema de bandas cambiarias, donde la tasa de deslizamiento de la paridad central fue establecida en 1,5\% mensual, conforme a la meta de inflación negociada con el FMI, con un movimiento del tipo de cambio de 290 bs $/ \$$ a 470 bs/\$ razón por la cual al cierre de 1996 la inflación se cifró en 103,2\% en tanto que la amplitud de la banda fue de $\pm 7,5 \%$ de acuerdo con el impacto promedio de los shocks en la cuenta corriente de la balanza de pagos y se estableció en 1,5\% mensual conforme al objetivo de la inflación.

El escenario antes descrito resultó en una disminución de la tasa de inflación del 103\% en 1996 al 20,1\% 
al finalizar 1999, pero con una contracción del PIBR del (7,2\%) y un déficit fiscal del 3,1\% del PIBR luego del superávit fiscal del 1,9\% del PIBR en 1997, pero con un saldo positivo de 5.465 millones de dólares en la cuenta corriente de la balanza de pagos según informes económicos del $\mathrm{BCV}$.

Cabe destacar que dicho sistema de bandas cambiarias se mantuvo hasta febrero de 2003, a pesar del ingreso de un nuevo gobierno en febrero de 1999, con una contracción del PIBR para el período 1997-2002 del 15\%, una tasa de inflación acumulada del 238,27\% según cifras del BCV (2013), con una cuenta corriente de la balanza de pagos superavitaria para un saldo acumulado de \$22.847 millones de dólares pero con un déficit fiscal promedio del 3,84\% del PIBR. Dicha situación fue consecuencia de que no se ponderaron los efectos devastadores sobre la industria y la agricultura al propiciar el auge de las importaciones y la liquidación de las exportaciones no petroleras.

La evidencia sugiere que no se valoraron adecuadamente los costos reales de una política antiinflacionaria, que reorientará la demanda hacia los bienes de origen importado, en detrimento de los producidos internamente, con su consiguiente incidencia sobre el PIBR, el empleo y su composición (Guerra, 2013). En este sentido, el manejo de la política cambiaria en Venezuela durante el esquema de bandas junio 1996febrero 2003, sugiere que la misma fue objeto de ataques especulativos precisamente porque no tuvo la flexibilidad necesaria para hacer frente tanto a los shocks reales como a los monetarios que suelen impactar a la economía venezolana, toda vez que se privilegió irrestrictamente el uso del tipo de cambio como único instrumento para el control de las presiones inflacionistas.

A partir de febrero de 2003, se asume nuevamente un control cambiario con una tasa de cambio de $1600 \mathrm{bs} / \$$. Desde entonces, debido al carácter expansivo de la política fiscal y monetaria, donde la política cambiaria de anclaje del tipo de cambio fijo como principal instrumento para abatir la inflación junto con los controles de precios, el producto interno bruto real en el período 2003-2014 ha crecido según cifras del BCV (2014) 54,21\%, mientras que la inflación acumulada ha sido de 1560,49\% con un saldo acumulado en la cuenta corriente en la balanza de pagos de 189.060 millones de dólares, producto de un crecimiento de más del $300 \%$ de los precios del petróleo, que pasaron de $\$ 25,76$ por barril a $\$ 103,46$ por barril al cierre de 2012 y \$88,42 al cierre de 2014, pero con un déficit fiscal del 5,51\% del PIBR en el año 2003 a 10,82\% en 2014.

Es importante recalcar que el control de cambios instaurado a partir del año 2003 ha sufrido varias devaluaciones a lo largo del período: de 1920 bs/\$ en 2004, 2150 bs/\$ en el período 2005-2009, 3260 bs/\$ en el año 2010, 4300 bs/\$ para el período 2011-2012 y 6300 bs/\$ para el período 2013-2014, ello sin contar las tasas de cambio SITME, SICAD I, SICAD II y recientemente SIMADI, lo cual ha llevado a que la econo- 
mía funcione hasta con 4 tasas de cambio, generando fuertes distorsiones en la estabilidad de precios y por consiguiente ante la reciente disminución de los precios del petróleo en más de un 54,37\%, una menor disponibilidad de divisas para importaciones y con ello una menor oferta de bienes transables para la economía lo cual se traduce en incremento general de los precios en promedio del 56,5\% en 2013 y 68,5\% en 2014 y se estima que para el 2015 la tasa de inflación no baje del 120\% (Econométrica y Ecoanalítica, 2015).

\section{Análisis de la relación entre el tipo de cambio implícito y el índice de precios al consumidor en} Venezuela período (1989-2014)

En la presente investigación se busca determinar la estrecha relación que existe entre la tasa de cambio implícita y el índice de precios al consumidor en Venezuela, la cual se calcula dividiendo el monto de la liquidez monetaria (M2) entre el saldo de las reservas internacionales, ambas variables reportadas por el Banco Central de Venezuela (BCV), mediante sus variaciones en los últimos 104 trimestres que comprenden el período sujeto a estudio, como se muestra en el Gráfico 1. Esto con la finalidad de prospectar el comportamiento de una en función mediante un análisis de regresión lineal simple, en aras de tener una proyección más precisa acerca de la evolución del índice de precios al consumidor y con ello de la tasa de inflación.

Para tales efectos se hace imprescindible que la función de regresión lineal cumpla con una serie característica de que le permitan ser aceptables sus resultados como se evidencia en el Gráfico 2, entre los que se encuentran una correlación positiva entre los valores pronosticados y los observados, que existan diferencias mínimas entre la suma de los cuadrados de los residuos y la suma de los cuadrados totales. De manera que la variabilidad del índice de precio observado está asociado al índice de precio pronosticado; así mismo se requiere que las variables sujetas a estudio presente una distribución normal de los datos y que los mismos se auto correlativos, para finalmente demostrar las condiciones de independencia, normalidad, colinealidad y homocedasticidad de la función mediante el análisis de los residuos.

Gráfico 1. Variación porcentual trimestral de la tasa de inflación y el tipo de cambio implícito 1989- 2014

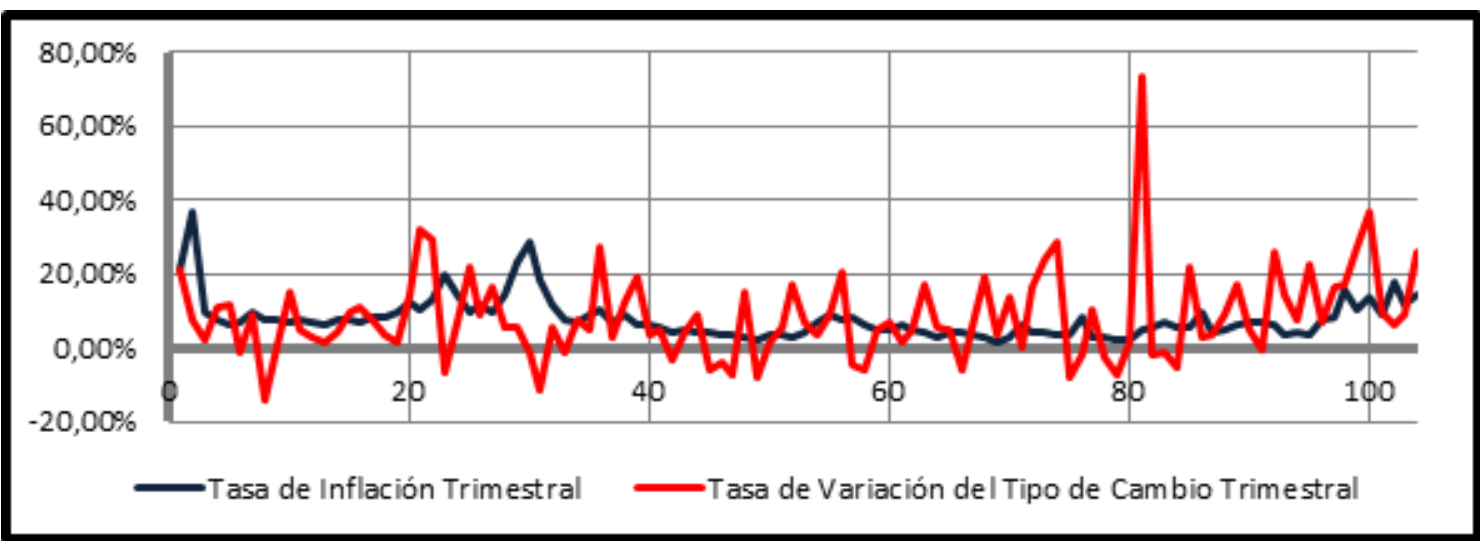

Fuente: Elaboración Propia en Base a Datos del BCV 1989-2014 
En el Gráfico 1 se observa la variación porcentual en los 104 trimestres comprendidos en el período 1989-2014, para las variables tasa de inflación trimestral y tasa de variación del tipo de cambio implícito, en donde se puede observar una oscilación muy similar entre ambas variables, sobre todo si se toma en cuenta que ambas variables presentan una variación promedio inter trimestral de 7,80\% para la tasa de inflación y $8,27 \%$ para la tasa de cambio implícito, con una correlación lineal de Pearson de 0,97 ; significando ello un alto de asociación entre la tasa de cambio implícita y el índice nacional de precios al consumidor, cuya relación se muestra en el gráfico ${ }^{\circ} 2$, donde en el eje de las ordenadas se tiene el índice trimestral de precios al consumidor y en el de las accisas la tasa de cambio implícita trimestral, dicho escenario antes descrito pudiese servir entonces para estimar el comportamiento de la tasa inflación a partir de la estimación del índice nacional de precios al consumidor en función del valor de la tasa de cambio implícita, mediante un análisis de regresión lineal.

Al mismo tiempo, en el Gráfico 2, se puede observar la ecuación de regresión $y=57,497 x+234,82$, la cual nos indica Y (Índice Trimestral De Precios al Consumidor) =234,82 +57,497X (tasa de cambio implícita). Así, según Anderson, Sweeney y Williams (2008), para analizar los coeficientes de regresión parcial debemos saber primero que cada valor de la tasa de cambio implícita le corresponde un pronóstico de índice trimestral basado en un incremento constante de 231,82 más 57,49 veces el valor de la tasa de cambio implícita, presentando esta función de regresión una correlación positiva perfecta de 0.966 entre el comportamiento del índice de precios observado con respecto al comportamiento de la tasa de cambio implícita y el índice de precios al consumidor pronosticado según la ecuación de regresión lineal, así mismo se muestra un $\mathrm{R}^{2}$ o coeficiente de determinación que no es más que el cuadrado del coeficiente de correlación de 0,933 , lo cual indica diferencias mínimas entre la suma de los cuadrados de los residuos y la suma de los cuadrados totales, concretamente, que el 93,3\% de la variabilidad del índice de precio observado está asociado al índice de precio pronosticado.

$$
R^{2}=1-\frac{\text { Suma_de_cuadrados_de_los_residuos }}{\text { Suma_de_los_cuadrados_totales }}
$$

En este mismo orden se observa un $\mathrm{R}^{2}$ corregido de 0,941 igual al $\mathrm{R}^{2}$ sin corregir, lo cual, según Anderson, Sweeney y Williams (2008), es una correlación a la baja en el número de casos y de variables independientes y este es estimado mediante la siguiente ecuación:

$$
R_{\text {corregido }}^{2}=R^{2}-\left[p\left(1-R^{2}\right) /(n-p-1)\right]
$$

En cuanto al estadístico F, que permite contrastar la hipótesis nula (Visuata, 2008), existe igualdad de 
medias y varianzas entre los valores observados de la variable independiente y los valores pronosticados por la ecuación de regresión lineal, donde $\mathrm{F}=\mathrm{t}^{2}$ donde $\mathrm{t}$ es el $\mathrm{t}$-Studens calculado para las dos muestra cuyo valor seria $t=\sqrt{F}$ siendo valor de $\mathrm{t}=37,81$ el cual es aceptable para unos grado de libertad 104-2=102 y un nivel de significación de 0,05 ; cuyo $t_{0,05}=1.658$, con lo cual el valor anterior del $t$ calculado demuestra que se encuentra en el área de probabilidad en el extremo superior de la distribución t por lo que las muestras son homogéneas es decir presentan una distribución normal de los datos.

Por otra parte, el estadístico de Durbin-Watson, utilizado para detectar la presencia de autocorrelación en una serie de datos y es una de las pruebas más utilizadas. Para este fin se define el estadístico de la siguiente manera:

Este estadístico de Durbin-Watson proporciona información sobre el grado de independencia o grado de autocorrelación existente entre los residuos de los valores observados con respecto a los valores pronosticados, donde $e_{i}=Y_{i} \square Y_{i}$. El estadístico Durbin-Watson oscila entre 0 y 4 , y toma el valor de 2 cuando los residuos son completamente independientes (Pardo y Ruiz, 2005). Los valores menores que 2, como es el caso en estudio, cuyo valor es de 0,129 indican una autocorrelación positiva entre el índice de precios al consumidor observado y pronosticado.

Gráfico 2. Índice nacional de precios al consumidor observado vs proyectado 1989 -2014

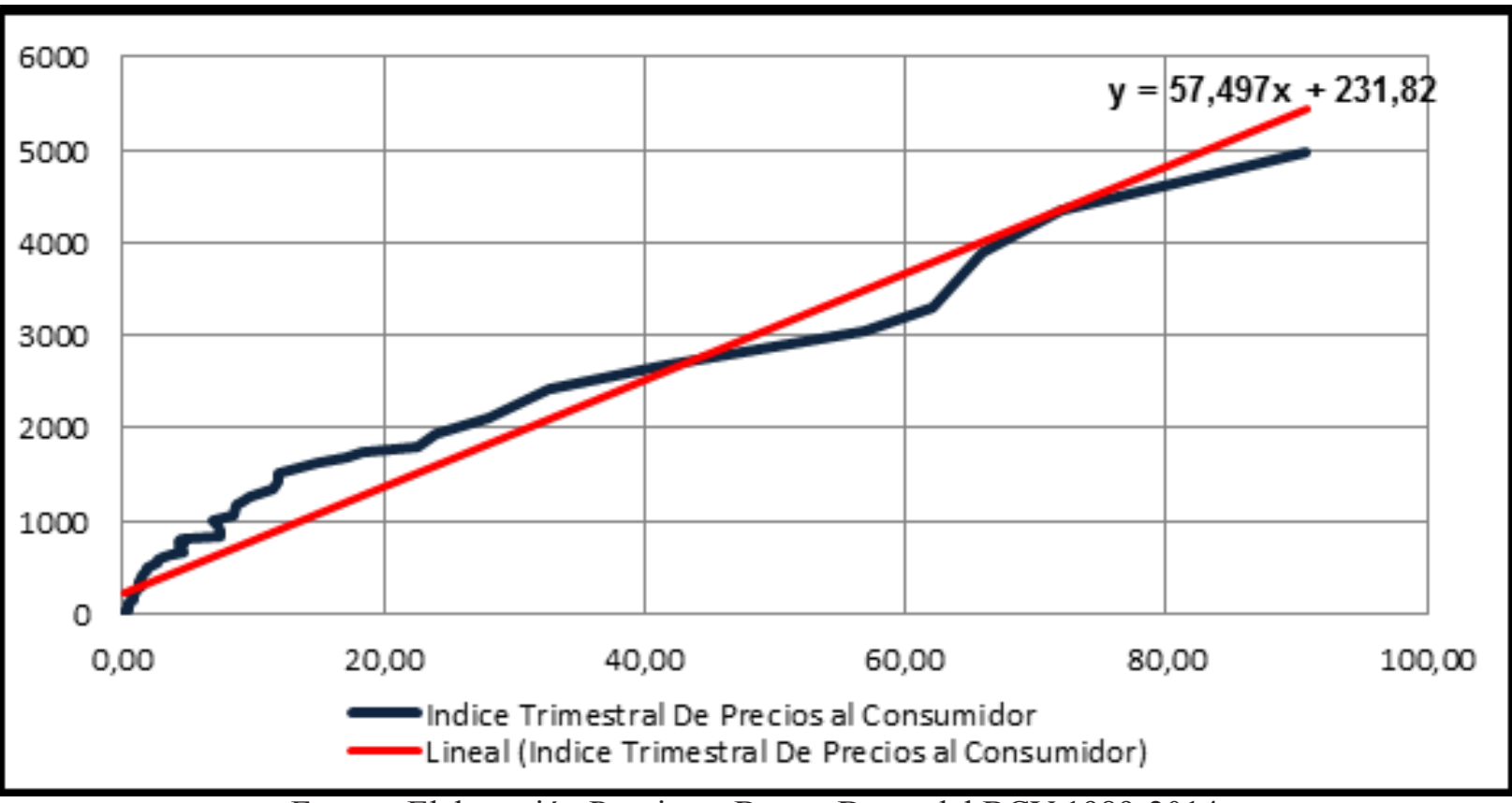

Fuente: Elaboración Propia en Base a Datos del BCV 1989-2014 
Al mismo tiempo se observa que el valor mínimo estimado por la ecuación de regresión lineal para el índice de precios al consumidor en función de la tasa de cambio implícita es de 234,69 y el valor máximo de 5447,377 cuya media para la índice de precios al consumidor es de 637,82, con una desviación típica \pm 919,685 con respecto a la media; mientras los residuos, es decir (diferencia entre los valores observados y los pronosticados), tienen un valor estimado mínimo de $-492,783$ y un valor máximo 612,786 cuya media es cero con una desviación típica $\pm 245,627$, todo lo cual indica una alta relación entre la variables observadas y pronosticada y una distribución normal en los residuos, presentando características de independencia normalidad, colinealidad y homocedasticidad.

Gráfico 3. Variaciones porcentuales anuales de reservas internacionales (RI), liquidez monetaria (M2) y producto interno real (PIBR) 1989-2014

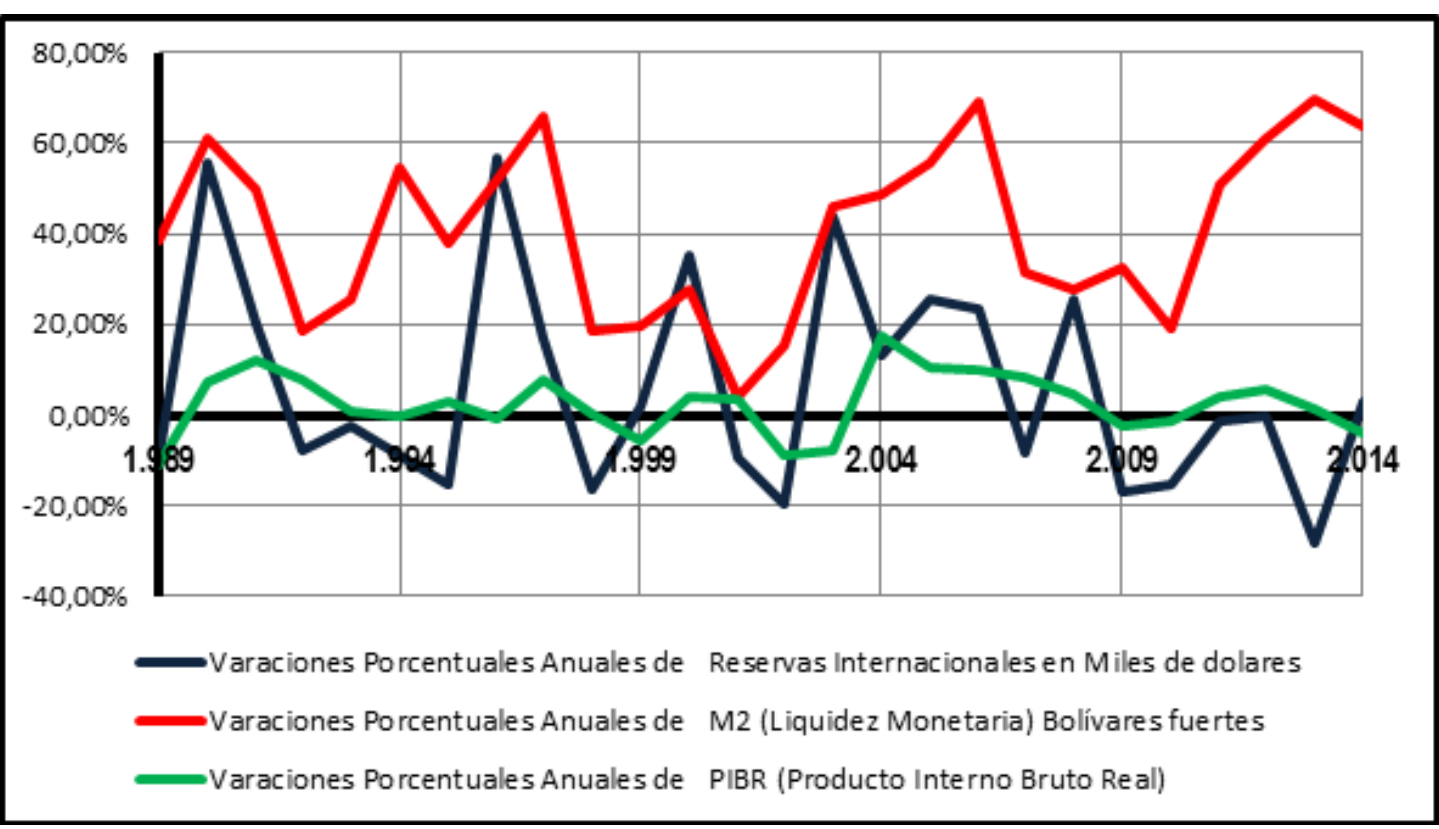

Fuente: Elaboración Propia en Base a Datos del BCV 1989-214

En el Gráfico 3 se observa el comportamiento de variables fundamentales que se toman como indicadores para manejar una política monetaria y cambiaría acertada lo es la liquidez monetaria, las reservas internacionales y el producto interno real si se parte de los postulados de la teoría cuantitativa del dinero la cual expresa que debe existir un equilibrio entre el crecimiento real de la economía y el aumento de la oferta monetaria hacia al mercado; así como también debe existir un principio de política monetaria fundamental que manejan los bancos centrales en los cuales la reservas internacionales una de sus funciones primordiales es la del respaldo del dinero circulante en aras de preservar la reserva de valor de la moneda.

En este sentido, en la economía venezolana existe una distopía en el comportamiento de las tres variables 
antes señaladas, con una variación anual promedio para el período 1989-2014 de 6,17\% para las reservas internacionales, 2,54\% para el producto interno bruto real y 40,98\% para la liquidez monetaria; al mismo tiempo se observa una baja correlación de la variación interanual de la liquidez monetaria con respecto a la variación interanual de la reservas internacionales cuyo valor es 0,36 ; así como también con la variación interanual del producto interno bruto real cuyo valor es 0,30 ; evidenciando un bajo grado de asociación entre las variables, lo cual es totalmente contrapuesto a lo que dicta la lógica de la política económica.

Estos escenarios antes planteados se traducen en una devaluación indirecta de la moneda porque la cantidad de bolívares en la economía crece más rápidamente que la cantidad de dólares que los respaldan en las reservas internacionales, produciendo una apreciación del dólar frente al bolívar y con ello un aumento de la tasa de cambio implícita, lo cual repercute en el incremento de precios de bienes importados.

Por otra parte, cuando la liquidez monetaria crece anualmente en promedio 16 veces más que el producto interno bruto real, se produce también un incremento de los precios de los bienes producidos nacionalmente debido a que la velocidad con la que se inyecta dinero a la economía es muy superior a la capacidad que esta tiene de absorber esa nueva masa monetaria mediante la producción de una mayor cantidad y diversidad de bienes, e impacta directamente eel comportamiento de los precios relativos haciéndolos inflexibles a la baja. Todo lo antes descrito repercute directamente en la capacidad del poder de compra del bolívar y por ende en el decrecimiento de los salarios reales de los trabajadores, a pesar de que los salarios nominales aumenten constantemente como se muestra en el Gráfico 4 en el caso del salario mínimo en Venezuela.

Gráfico 4. Salario mínimo nominal vs salario mínimo real 1989-2014

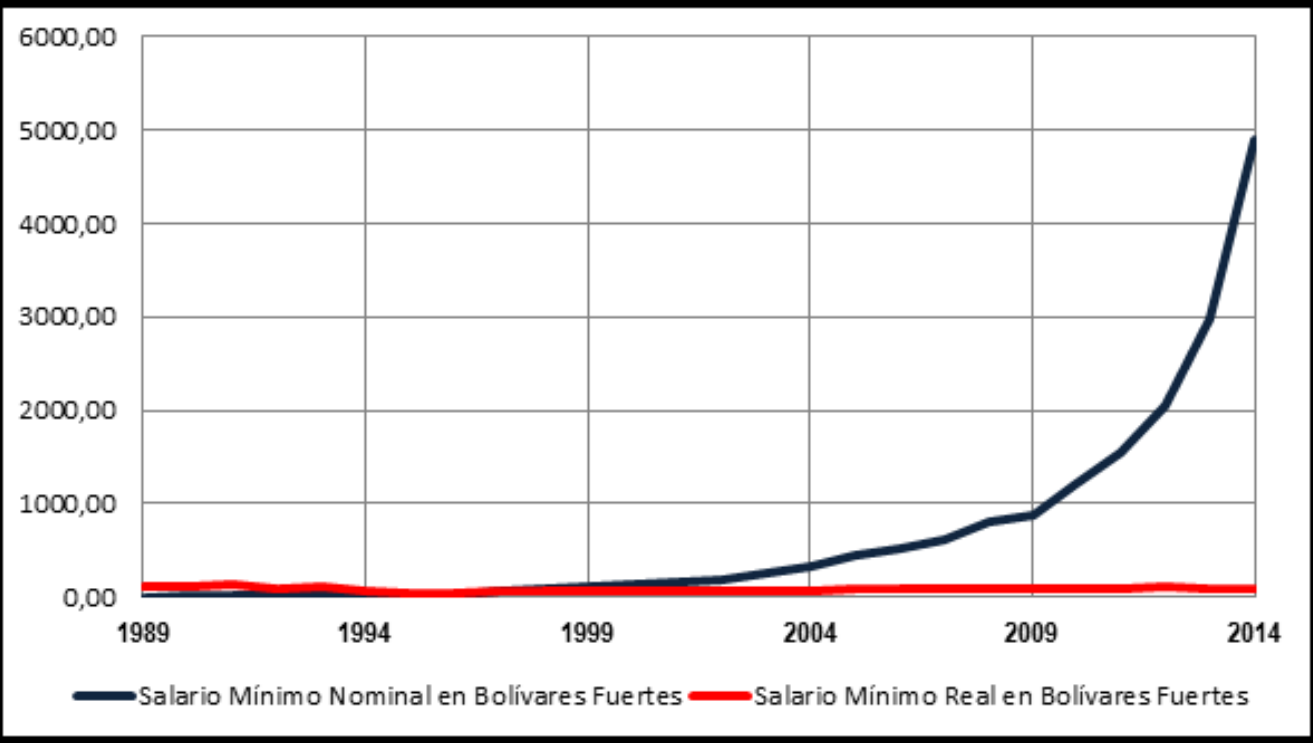

Fuente: Elaboración Propia en Base a Datos del BCV 1989-2014 
En el Gráfico 4 puede evidenciarse como en el período 1989-2001 existe un comportamiento muy similar entre valor del salario real y nominal, sin embargo a partir del año 2002 como consecuencia de un política monetaria expansiva lo cual repercutió en el incremento general precios tanto de los bienes importados como de producción nacional esto se tradujo en la creación de una brecha entre los salarios nominales y reales, dando como resultado que el salario mínimo en Venezuela desde el año 2003 no cubra el valor de la canasta básica y desde el año 2008 no cubra el costo de la canasta alimentaria para un grupo familiar de 2 personas tal como se evidencia en el gráfico $n^{\circ} 5$.

\section{Gráfico 5. Costo de la canasta básica familiar, canasta alimentaria y salario mínimo nominal}

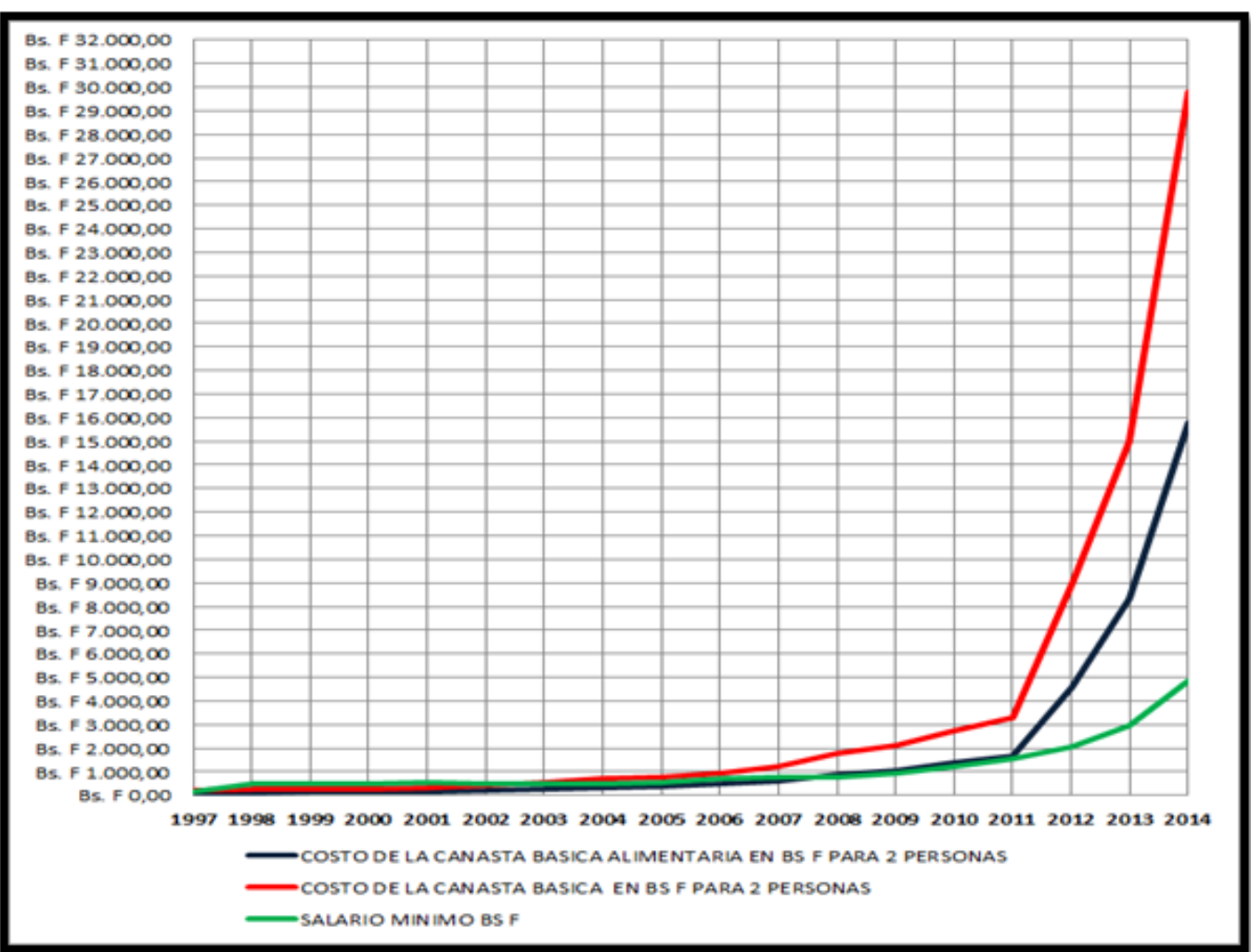

Fuente: Elaboración Propia en Base a Datos del CENDAS-FVM 1997-2014

Ahora bien, aun cuando en los últimos 12 años, en la economía venezolana la velocidad con la que aumenta la liquidez monetaria es muy superior a la rapidez con la que crece la renta real y esto produce un aumento de los precios producidos localmente, no es menos cierto que ello tiene una importante incidencia en la tasa de cambio implícita porque el crecimiento de la liquidez monetaria es superior al nivel en el que crecen las reservas internacionales, traduciéndose ello en una devaluación no oficial de nuestro signo monetario y con ello la pérdida de su función como reserva de valor lo cual estimula la salida de capitales registrados y no registrados a través de la balanza de pagos, produciendo preocupantes desequilibrios en el 
saldo de la misma, pues, tal situación repercute en la constante pérdida de activos en reservas internacionales, tal como se muestra en el Gráfico 6. \$)

\section{Gráfico 6. Saldo en Transacciones Corrientes de Capital y Financiera Y Errores y Omisiones en (Millones de US}

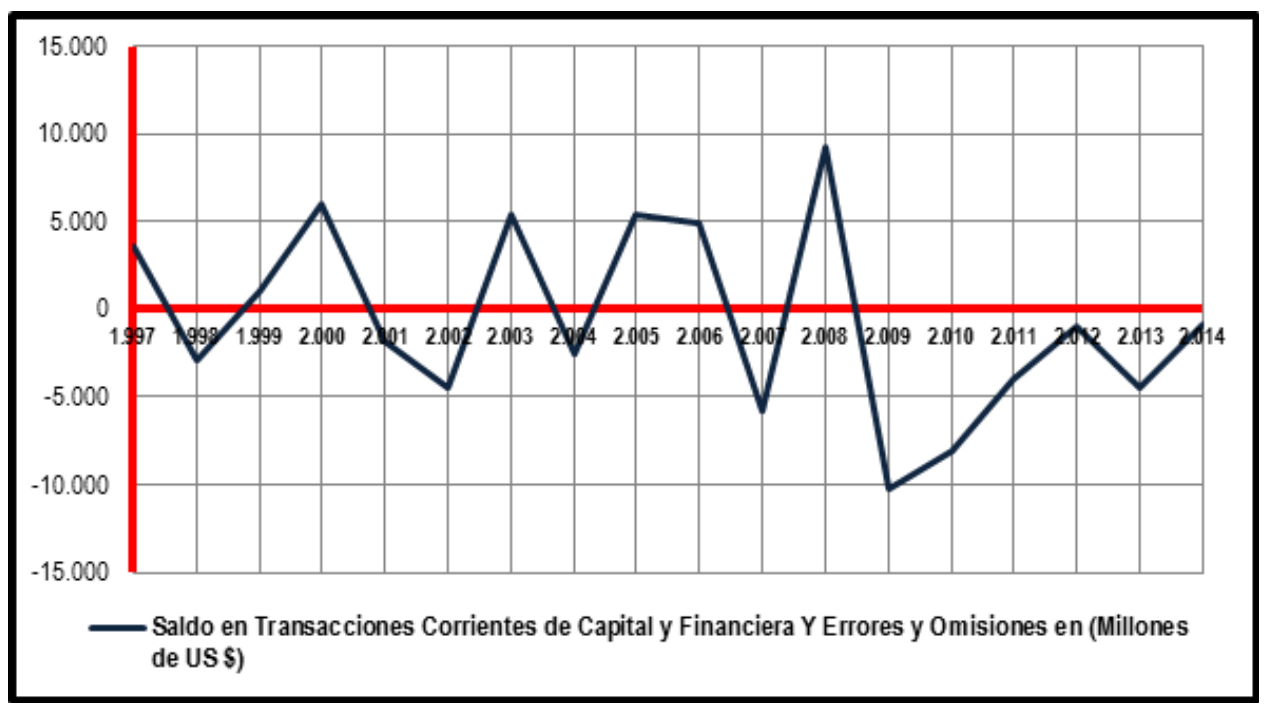

Fuente: Elaboración Propia en Base a Datos del BCV 1997-2014

Finalmente, puede concluirse luego del presente análisis que las variaciones desproporcionadas entre la liquidez monetaria y el nivel de reservas internacionales tiene un impacto directo en el aumento de la tasa de cambio implícita y ello a su vez guarda una relación directa con el incremento del índice de precios al consumidor, lo cual repercute, en últimas, en la tasa de inflación anualizada, debido a que la mayor parte de los bienes que consume la economía venezolana son importados.

\section{CONCLUSIONES}

En la presente investigación se evidencia alto grado de asociación entre las variables observadas y pronosticadas y una distribución normal en los residuos, las cuales muestran un grado de correlación positiva alta entre el comportamiento del precio observado según el valor de la tasa de cambio implícita y el índice de precio al consumidor predicho según la ecuación de regresión lineal aplicada. Ello muestra que casi en su totalidad la variabilidad del índice de precio al consumidor observado está asociado al índice de precio al consumidor predicho.

El estadístico F permitió contrastar la hipótesis nula, donde existe igualdad de medias y varianzas entre los valores observados de la variable dependiente y los pronosticados por la ecuación de regresión lineal, 
mostrando una autocorrelación positiva, donde podemos visualizar una relación directa entre la tasa de cambio implícita y el índice de precio al consumidor, lo cual presenta características de independencia normalidad, colinealidad y homocedasticidad.

Finalmente, podemos decir que la escogencia del régimen cambiario más apropiado para una economía es una asignación de por sí difícil. La complejidad aumenta al considerar los rasgos particulares de una economía petrolera como la venezolana, en la que los cambios de signo en los términos de intercambio siempre están en la agenda de la política económica. Esos shocks reales que suelen acompañar a Venezuela no se disipan con rapidez sino que más bien tienden a traducirse en perturbaciones fiscales y monetarias en virtud del particular mecanismo de conformación de la estructura fiscal y de los mecanismos de creación y destrucción de dinero.

La valoración del período de tipo de cambio fijo a partir de febrero de 2003 apunta a que la economía mostró un deficiente desempeño durante parte importante de ese lapso, en virtud de que una política fiscal expansiva no respalda el anclaje del tipo de cambio. Por esta razón, las cuentas fiscales perdieron la senda del equilibrio que las había caracterizado, el esquema de fijación del tipo de cambio comenzó a confrontar problemas de credibilidad toda vez que la inflación se aceleró, y el tipo de cambio real se apreció y se produjo fuga de capitales.

Al final, ello se tradujo en un ataque especulativo que terminó generando un proceso de recesión económica con hiperinflación para la economía venezolana. De la experiencia analizada, es claro que el tono de la política fiscal ha determinado los resultados esperados de los distintos arreglos cambiarios instrumentados en Venezuela. Ante una actuación fiscal que no apoya la reducción de la inflación, el esfuerzo de la política monetaria y cambiaria se magnifica, lo que no necesariamente resulta conveniente para la solvencia del sistema financiero o la viabilidad del sector externo.

\section{REFERENCIAS}

Anderson, D., Sweeney, D. y Williams, T (2008). Métodos cuantitativos para los negocios. México: Editorial Thomson. Novena edición.

Blanchard, O. Enrri D (2006). Macroeconomía. México: Prentice Hall.

Chávez Alizo, Nilda (2001). Introducción a la investigación educativa. Servicio Autónomo de Imprenta del Estado Zulia, Venezuela.

Ekelund, R. Hébert R. (2008). Historia de la teoría económica y de su método. Cuarta edición. Mexico: Editorial MC Graw Hill. . 
Fernández, A., Rodríguez, L. (2008) Política económica. México: Editorial McGraw-Hill.

Guerra, José. (2013). El legado de Chávez. Un análisis de la economía venezolana y sus posibilidades. Venezuela: Editorial Libros X Marcados.

Guerra, José, Pineda, Julio (2000). Trayectoria de la política cambiaria en Venezuela. Vicepresidencia de Estudios del Banco Central de Venezuela. Venezuela.

Hernández, Fernández y Baptista (2008). Metodología de la investigación. México: Editorial McGraw-Hill. Herrerías, A. (2011). Historia del pensamiento económico. México: Editorial LIMUSA.

Martínez M., (2013). Epistemología y metodología cualitativa en las ciencias sociales. Colombia: Trillas. . Mochón F. (2008). Introducción a la macroeconomía. México: Editorial McGraw-Hill.

Pardo Antonio y Ruiz Miguel (2005). Análisis de datos con SPSS 13Base. México: Editorial MC Graw Hill. Visuata, Bienvenido (2008). Análisis de estadístico con SPSS 14 Estadística Básica. México: Editorial MC Graw Hill.

\section{Referencias Electrónicas}

BCV (Banco Central de Venezuela), http://www.BCV.org. Consulta 07/03/2015

BCV (Banco Central de Venezuela), http://www.bcv.org.ve/c2/indicadores.asp. Consulta 09/03/2015

BCV (Banco Central de Venezuela), http://www.bcv.org.ve/c1/abceconomico.asp. Consulta 10/03/2015

BCV (Banco Central de Venezuela), http://www.bcv.org.ve/cuadros/2/212a.asp?id=64. Consulta 10/03/2015

CENDAS-FVM (Centro de Documentación y Análisis Social de la Federación Venezolana de Maestros) http://www.cendasfvm.org/. Consulta 06/03/2015

ECONOMÉTRICA.http://www.econometrica.com.ve/blog/category/inflacion/. Consulta 06/03/2015.

ECOANALÍTICA.http://www.ecoanalitica.net/newsite/spanish/site/p_contenido.php?sec=10 $\&$ cont $=4 \&$ cat $=9 \&$ tcat $=4.06 / 03 / 2015$. 